\title{
THE USE OF STABLE ISOTOPES IN BIOLOGICAL AND MEDICAL RESEARCH
}

\author{
BY IRVING M. LONDON
}

(From the Department of Medicine, College of Physicians and Surgeons, Columbia University, and The Presbyterian Hospital in the City of New York)

During the past 15 years there has been a rapid and extensive development of the use of isotopes in biological and medical investigation. In 1923, Hevesy's pioneering studies, in which thorium B was used to investigate the absorption and localization of lead by plants, revealed some of the possibilities of the use of tracer technique for biochemical research. The exploitation of these possibilities marked time, however, until isotopes of elements more widely associated with biochemical processes were made available. The discovery of deuterium by Urey in 1931 and the development of methods for production of heavy water were the first steps toward providing stable isotopes for tracer studies in biological systems.

There soon followed the preparation of the heavy isotopes of nitrogen, carbon and oxygen, which, with hydrogen, constitute a large portion of the elements of interest to the biologist. The discovery of artificial radioactivity by Joliot and Curie in 1934 led before long to the production of a number of radioactive isotopes which are of value in biological investigations. The past decade and a half have witnessed a swift and enthusiastic application of stable and radioactive isotopes to a wide variety of biologic studies.

The reports of these studies form a voluminous literature. Several reviews (1-7) which deal with various aspects of tracer research have appeared in recent years. The scope of the present discussion is limited to the use of stable isotopes and it is not the purpose of this paper to attempt another review, which would require far more space than is available. This paper is designed to indicate some of the types of investigative problems for which tracers are particularly suited and to present illustrative examples of studies in which stable isotopes have been used.

The discussion is presented in two parts. I. Types of problems for which isotope investigation is suitable. $A$. Problems of dilution and transport-the addition of isotopic substance A to a biologic system and the subsequent isolation of substance $A$ and the determination of its isotope content. $B$. Problems of conversion-the addition of isotopic substance $A$ to a biologic system and the subsequent isolation of substance $B$ and the determination of its isotope content. $C$. Kinetics of biochemical reactions. II. An illustration of the use of isotope techniques in the investigation of related problems in one field: the biosynthesis and metabolism of porphyrins.

\section{Problems of Dilution and Transport}

Shortly after Hevesy's first experiments with thorium B, one of his colleagues expressed the hope that a tracer would be found which could permit the determination of the fate of individual water molecules such as those in the tea they were about to drink (8). Within a decade Urey's discovery of deuterium and preparation of heavy water had provided an indicator which could help in the solution of such a problem. This type of problem is characteristic of a number of questions which can be answered by a basically simple application of the isotope technique. This application consists in introducing a labeled substance of known isotope concentration into a system and subsequently measuring the isotope concentration of the same substance in the system as a whole or in one or more of its constituent units. One can trace the fate of the labeled substance, i.e., its distribution and localization, and one can measure the time relationships of its distribution. In terms of biological experimentation, this technique is applicable to the study of mechanisms and rates of transfer of biological substances by absorption, secretion or excretion and to the study of diffusion and membrane permeability. The same technique is applicable to the solution of quantitative analytic problems, whether primarily biological or chemical. By determining the dilution in isotope concentration of the labeled substance after its addition to the system one can determine the amount of this substance which is present in the system. In the animal organism precise measurements of a variety of body constituents are made possible, and in a purely chemical system, 
the isotope dilution method affords a precise technique for quantitative analysis.

A few examples which are representative of the various applications of this type of tracer work may serve to illustrate the use of the technique. Hevesy and Hofer investigated the rate at which water is eliminated from the human body (8). They administered heavy water to a subject and subsequently measured the deuterium concentration in the urine. By determining the interval between the time of maximal concentration of deuterium in the urine and the time when the deuterium concentration in the urine was one-half the maximal value, they determined $t_{1 / 2}$, the time required for one-half of the heavy water ingested to be eliminated from the body. This value is nine days. Inasmuch as the biologic behavior of heavy water of low isotope concentration is essentially the same as that of ordinary water, this value for $t_{1 / 2}$ applies equally to ordinary light water. The average time which a water molecule spends in the body is $t_{1 / 2} \times \ln 2$, or 13 days.

In the same study, these investigators applied the principle of isotope dilution to determine the water content of the body. After the first day the deuterium concentration in the water of urine may be taken as the deuterium concentration in total body water. This is based on the reasonable assumption that the heavy water becomes completely mixed with the total body water and is handled in the body in a manner indistinguishable from that of ordinary water. The total body water volume is then equal to the product of the deuterium concentration and the volume of the heavy water administered divided by the deuterium concentration in the urine. These calculations yielded a value for total water volume of $43 \pm 3$ liters or $63 \pm 4$ per cent of the body weight. This method was modified by Moore (9) who determined the deuterium concentration in plasma one hour after the intravenous injection of heavy water. Complete equilibration of the injected $\mathrm{D}_{2} \mathrm{O}$ with the body water was found to occur within one hour; consequently the deuterium concentration in the one-hour plasma sample could be considered equivalent to the deuterium concentration in total body water. By this method, a value for total body water of 47.8 liters or 72 per cent of the body weight was obtained in a normal adult male.

The ability of the tracer technique to follow the transfer of a substance across a membrane has permitted the study of a number of biological problems which could not be satisfactorily investigated by other methods. Without the aid of isotopes, the measurement of such transfer would depend on the determination of the net change in the quantity of the substance on each side of the membrane. Under physiological conditions, however, little or no net change in the quantity of a great many substances is found despite considerable transfer, for the transfer takes place simultaneously in both directions across the membrane. The isotope technique avoids this difficulty. By introducing a labeled substance on one side of the membrane one can follow the movement of the labeled substance in a system at equilibrium or in a steady state. Inasmuch as the biochemical behavior of labeled units is indistinguishable from that of unlabeled units of the same substance, the transfer of the labeled substance is representative of the transfer of the substance as a whole.

The studies of Gellhorn and Flexner (10) on the transfer of water across the placenta illustrate this application of the isotope technique. $\mathrm{D}_{22} \mathrm{O}$ was injected into a pregnant guinea pig, and after ten minutes during which equilibration of $\mathrm{D}_{2} \mathrm{O}$ between the various fluid compartments of the mother was known to occur, the fetuses were delivered by Caesarean section. By determining the total body water of the fetus and the deuterium concentrations in maternal and fetal plasma, it was possible to calculate the quantity of water transferred across the placenta per unit time. It was found that the rate of transfer of water across a unit weight of placenta from the 28th day of gestation to term increased about nine times, and that the rate of tranfer of water to a unit weight of fetus was parallel to the relative growth rate of the fetus.

The principle of isotope dilution has found ready application to quantitative analytic studies (11-13). Most commonly, a small known amount of labeled substance is added to a system containing an unknown amount of the same substance unlabeled. By determining the dilution in the isotope concentration of the substance isolated from the system, the quantity of substance originally present in the system can be calculated. This technique permits accurate quantitative determinations of a large number of substances for which other good quantitative methods may not be available. In addition, the need for quantitative isola- 
tion of the substance is obviated. A pure sample adequate in amount for isotope analysis is sufficient. For accurate results it is essential that the labeled material which is added to the system and the material isolated from the system be pure.

The isotope dilution method has been used to determine quantitatively the optical isomers of amino acids present in amino acid mixtures (14) and to determine the amino acid content of horse hemoglobin (15) and human and bovine albumin (16). Its use has demonstrated that the claim for the presence of large quantities of D-glutamic acid in malignant tumor tissue is invalid (17).

A modification of this procedure has been described by. Keston, Udenfriend, and Cannan (18) for amino acid analyses. A mixture of amino acids is treated with a labeled substance, e.g., piodophenylsulfonyl chloride labeled with $\mathrm{I}^{131}$. A large amount of the non-isotopic p-iodophenylsulfonyl derivative of the amino acid to be measured is added to the mixture and the compound is subsequently isolated and its specific activity (isotope concentration in the case of stable isotopes) determined. Inasmuch as stable derivatives may be formed with other amino acids in the mixture, the isolated compound may require numerous recrystallizations before purity is established. It should be noted that complete conversion of the amino acid to its derivative must occur if accurate results are to be obtained. This method permits the quantitative determination of microgram quantities of amino acids.

The principle of isotope dilution has been applied in reverse by Bloch and Anker (19) to determine the isotope concentration of a metabolite present in too small an amount to permit direct isolation. Since the amount of isotopic metabolite is also unknown, the addition of a known amount of nonisotopic metabolite (carrier) merely facilitates isolation, but calculation of the original isotope concentration of the metabolite is not possible. If two different quantities of normal carrier are added to separate aliquots of the metabolite solution, however, the two samples of metabolite subsequently isolated will have different isotope concentrations. Sufficient data are then available for calculation of the original isotope concentration. The error of this procedure, however, may be as high as 20 per cent.

\section{Problems of Conversion}

Isotopes are particularly suited to the study of conversion reactions. One of the principal types of investigation in intermediary metabolism is concerned with determining the products of the biological conversion of a substance or to determine its biologic precursors. Experiments performed without the aid of isotopes are for the most part of two types: (1) in vivo studies in which substance $\mathrm{A}$ is administered and the quantity of substance $\mathrm{B}$ which is formed in the tissues or is excreted is determined; and (2) in vitro studies in which substance $\mathrm{A}$ is added to tissue slices or isolated organs and the quantity of substance $B$ formed in the system is measured. Although these types of study have yielded much valuable information, they do not yield conclusive evidence for the determination of biologic precursors or conversion products. In vivo studies in which an increased excretion of substance $B$ following the administration of substance $A$ is interpreted as evidence that $A$ is a biologic precursor of $\mathrm{B}$, are open to the criticism that increased excretion of $B$ represents an increased loss of $\mathrm{B}$ from the organism rather than increased synthesis and that $A$ is responsible for this effect merely by virtue of its effect on the excretory mechanism. An increased formation of $\mathrm{B}$ in vivo or in vitro following the addition of $\mathrm{A}$ does not prove the conversion of $A$ to $B$ but may indicate rather that $\mathrm{A}$ is concerned in reactions yielding energy required for the synthesis of B. Furthermore, the absence of increased formation of $\mathrm{B}$ following the administration of $\mathrm{A}$ does not preclude the role of $\mathrm{A}$ as a specific precursor for $\mathrm{B}$. Most biologic syntheses involve a series of reactions. Only one of these reactions may be limiting and principally responsible for the rate of formation of B. If the specific conversion of precursor A to substance $B$ is not this limiting reaction, the administration of $A$ will probably not be reflected by an increased formation of B. Clearly another technique of investigation which offers unequivocal evidence for the specific utilization of structural components of one substance for the biologic synthesis of another is required. Such is the isotope technique.

A striking example of the use of isotopes in the study of conversion reactions is the investigation of the biological synthesis of creatine and creatinine. Bloch and Schoenheimer (20) addressed their attention first to the relationship in vivo of 
creatine to its anhydride, creatinine. Creatine labeled with $\mathrm{N}^{15}$ was fed to rats and subsequently creatine was isolated from the muscles and creatinine from the urine. The $\mathrm{N}^{15}$ concentrations in the isolated creatine and creatinine were nearly identical and indicated that urinary creatinine is derived from body creatine. Similar studies were carried out in which after the preliminary period of feeding $\mathrm{N}^{15}$ labeled creatine, the rats were kept on. a creatine-free diet, during which time the isotope content of urinary creatinine was iclentical with that of body creatine. This finding indicated that body creatine is the sole precursor of urinary creatinine in an animal on a creatine-free diet. Were any other nitrogenous source present, the isotope concentration in the creatinine would have been lower than that of the body creatine as a result of dilution with non-isotopic nitrogen.

To determine whether the creatine $\rightleftarrows$ creatinine reaction is reversible in vivo as it is in vitro, isotopic creatinine was administered to rats and body creatine was subsequently isolated. There was essentially no isotopic nitrogen in the creatine, a finding which indicates that in vivo creatinine is not convertible to creatine.

The next step in these studies was the search for the biologic precursors of creatine. Clues to the identity of these precursors were provided by Brand et al. $(21,22)$ who found that patients with muscular dystrophy excrete more creatine after administration of glycine, and by Fisher and Wilhelmi (23) who reported that the addition of arginine to the fluid perfusing rabbit hearts resulted in a quantitative conversion to creatine. In addition Borsook and Dubnoff showed that guanidoacetic acid is methylated to creatine in surviving liver slices and that this methylation is markedly accelerated by the addition of methionine; they concluded that the methyl group of methionine is transferred to guanidoacetic acid to form creatine (24). Simultaneous and independent investigations by Bloch and Schoenheimer revealed that when glycine labeled with
$\mathrm{N}^{15}$ was administered to normal rats, creatine with a high isotope content was formed (25). Similarly sarcosine labeled with $\mathrm{N}^{15}$ yielded creatine with equally high isotope content. Inasmuch as the ingestion of isotopic sarcosine was shown to lead to the deposition in proteins of isotopic glycine to the same extent as when glycine itself was fed, it was concluded that sarcosine is very rapidly demethylated after its ingestion and that it serves as a precursor of creatine only after demethylation to glycine (26). When guanidoacetic acid labeled with $\mathrm{N}^{15}$ was fed, creatine was formed with an isotope content approximately the same as when an equivalent amount of isotopic creatine was administered; clearly, methylation of guanidoacetic acid to form creatine must occur quickly (25).

With the role of glycine as a precursor established and with the demonstration of the rapid methylation of guanidoacetic acid to form creatine, there yet remained the problem of determining conclusively the biologic sources of the various groups in the creatine molecule. Working independently, Borsook and Dubnoff found that kidney slices could form guanidoacetic acid from arginine and glycine (27) ând Bloch and Schoenheimer showed that the administration of arginine labeled with $\mathrm{N}^{15}$ in the amidine group resulted in the formation of creatine of high isotope concentration (28). By degradation studies the latter investigators were able to show that the amidine nitrogen of creatine is derived from the amidine nitrogen of arginine, and that the nitrogen of the sarcosine group originates from glycine (29).

The final link was provided when du Vigneaud et al. found that the administration to rats of methionine labeled with deuterium in the methyl group results in the formation of creatine with a high deuterium concentration $(30,31)$.

These studies established the immediate biological source of each part of the creatine molecule. The biosynthesis of creatine may be summarized as follows:

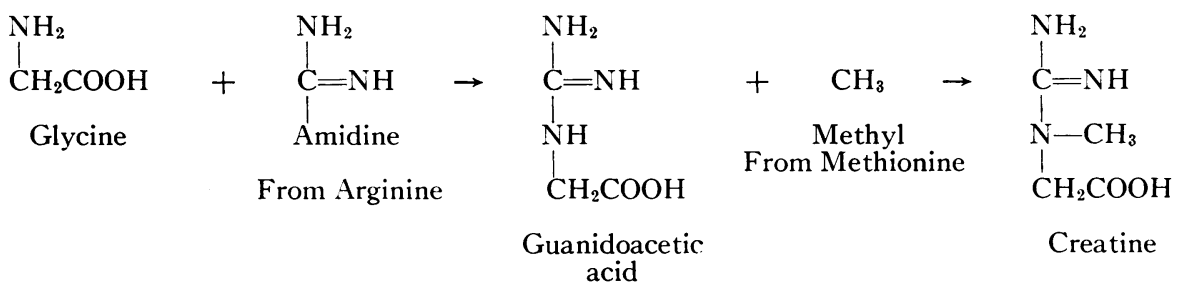


Recent studies on the biosynthesis of uric acid offer another example of the use of the isotope technique in establishing the identity of the biologic precursors of an important chemical substance. Sonne, Buchanan and Delluva (32-34) prepared a number of compounds labeled with $\mathrm{C}^{\mathbf{1 3}}$, administered them to pigeons, isolated the uric acid from the excreta, and degraded the uric acid by procedures which permitted separate isotope analyses of each of the carbon atoms in the uric acid molecule. They found that the number 6 carbon is derived from $\mathrm{CO}_{2}$, carbon atoms 2 and 8 from the carboxyl carbon of acetate $\left(\mathrm{CH}_{3} \mathrm{C} * \mathrm{OOH}\right)$ or formate $\left(\mathrm{HC}^{*} \mathrm{OOH}\right)$, and carbon atom 4 from the carboxyl carbon of glycine $\left(\mathrm{CH}_{2} \mathrm{NH}_{2} \mathrm{C} * \mathrm{OOH}\right)$. Shemin and Rittenberg (35) demonstrated the specific utilization of the amino group of glycine for nitrogen 7 in an experiment in which $\mathrm{N}^{15}$ labeled glycine was fed to a human and the uric acid isolated from his urine was degraded. $\mathrm{Ni}$ trogen atoms 1,3 and 9 are apparently derived from ammonia of the general metabolic pool. With glycine established in positions 4 and 7 , it remained for Karlsson and Barker (36) using glycine labeled with $\mathrm{C}^{14}$ in the $\alpha$ position to show that the methylene carbon of glycine is incorporated into position $5\left(\mathrm{NH}_{2} \mathrm{C} * \mathrm{H}_{2} \mathrm{COOH}\right)$. These composite data derived from both avian and human experiments have established the immediate biologic precursors of each component of the uric acid molecule.

Progress in the delineation of the conversion reactions in intermediary metabolism has been markedly facilitated by the use of isotopes. Limitations of space prevent discussion of the many excellent studies which illustrate this application of tracer technique. The interested reader is referred to reviews or representative papers by Wood (37), Evans (38), Gurin (39), Stetten (40), Weinhouse (41) and their colleagues for a more thorough consideration of the use of tracers in the study of conversion reactions.

\section{Kinetics of Biochemical Reactions}

Within a few years after the start of their investigations in intermediary metabolism with the aid of isotopes, Schoenheimer, Rittenberg and their colleagues concluded that the conventional concept of the division of metabolic processes into exogenous and endogenous forms was untenable.
Their studies demonstrated that there is a constant interchange of dietary constituents with body constituents and that the body constituents are in a state of flux, of continuous synthesis, degradation, and interchange. They termed this concept of intermediary metabolism "the dynamic state of body constituents." This concept not only comprises an appreciation of the interconversions and synthetic and degradative reactions of body constituents, but it emphasizes the importance of the rates at which these reactions are carried out in the living organism. The kinetics of processes in intermediary metabolism has received relatively little attention, however, as compared with the attention focussed on delineating the pathways of synthesis and degradation. In the future, however, the kinetic aspects of biologic processes may be expected to receive considerably more emphasis in isotope studies. An appreciation of the importance of a metabolic road requires a knowledge of the extent of the traffic on that road. When more than one pathway for the conversion of one substance to another exists, the rates at which the reaction is carried out via the different routes indicate the relative significance of these routes.

Studies in reaction kinetics are pertinent to the investigation of chemical changes in biological fluids and tissues which occur in disease. By determining reaction rates, it is possible to ascertain whether an abnormally high concentration of a substance is the result of an increased rate of synthesis or a diminished rate of degradation, and whether an abnormally low concentration results from a diminished rate of synthesis or an increased rate of degradation.

The considerations and calculations involved in the measurement of reaction rates in isotope experiments have been discussed by Zilversmit et al. (42), by Branson (43), and by Radin (44). The principal consideration is the basic assumption of biological isotope research, namely, that isotopic molecules are biologically indistinguishable from non-isotopic molecules. In nearly all cases the additional assumption may be made that the appearance and disappearance of all molecules proceed at random. The simplest type of problem of reaction kinetics is concerned with a biological system in the steady state, i.e., the rate of appearance of the substance under study equals its rate of disappearance and, during the period of study, these rates remain constant. Under these conditions, the total number of molecules, $M$, of the substance in the system is equal to $m$, the number of molecules which enter (or leave) the system per unit 
of time, multiplied by the turnover time, $\overline{\mathrm{T}}$, the time required for the appearance in the system or disappearance from the system of $\mathrm{M}$ molecules of the substance.

One type of experiment in which the rate of synthesis and degradation of a substance may be calculated is that in which a small amount of the labeled substance which is normally synthesized in the system is added to the system and its rate of disappearance is measured. In the steady state the rate of disappearance is equal to the rate of appearance and is consequently a measure of the rate of turnover. The rate of creatine turnover was studied by this technique (45). Adult rats were given isotopic creatine and were then placed on a creatine-free diet. Samples of creatinine were isolated at intervals from the urine. The isotope content of the creatinine was shown in the studies described earlier to be the same as that of the body creatine. The decrease in isotope concentration in the urinary creatinine indicates therefore the disappearance of labeled creatine from the tissues and its replacement by newly synthesized nonisotopic creatine. The curve of isotope concentration in the creatinine declines in exponential fashion, i.e., the decline in isotope concentration at any moment is proportional to the isotope concentration at that moment. Since the slope, $\mathrm{K}$, of the declining isotope concentration curve is equal to $\frac{m}{M}$, i.e., the fraction of the total substance $M$ which disappears from the system per unit time, and since $i$ is the isotope concentration at any time $t$, then $\frac{d i}{d t}=-k i$.

On integration $i=i_{0} e^{-k t}$, in which $i_{0}$ is the isotope concentration at the start of the experiment. On converting to $\operatorname{logarithmic}$ values, $\ln i=\ln i_{0}-k$ t. A plot of $\ln i$ against $t$ yields a straight line whose slope is $k$. Accordingly, by serial determinations of the isotope concentration, $k$ can be calculated, and $t_{1 / 2}$, the half lifetime, can be determined from the equation $t_{1 / 2}=\frac{\ln 2}{k}$. Since $\bar{T}=t_{1 / 2} \times \ln 2$, the turnover time is also readily calculated. To express the rate of turnover in terms of units of weight per unit of time, $M$ must be determined quantitatively by an independent procedure.

In the case of creatine in rats, the turnover rate was found to be about 0.02 per day. By determining the total body creatine, and the daily urinary creatinine excretion, it was found that the daily creatinine excretion corresponds to about 2 per cent of the total body creatine. The close agreement of these values for daily synthesis of creatine and excretion of creatinine precludes the existence of any major catabolic pathway for creatine other than creatinine formation and excretion.

Hoberman et al. $(46,47)$ have utilized this technique to study the rate of turnover of body creatine in man. In two human male subjects on a creatine-free diet values of 42 and 48 days for the half lifetime of body creatine were obtained $\left(t_{1 / 2}=\frac{\ln 2}{k}=\frac{0.69}{0.0164}=42 ; \frac{0.69}{0.0143}=48\right)$. When methyl testosterone is administered to the normal subject, the slope of the curve becomes steeper and reflects a more rapid dilution of body creatine (47). By appropriate calculations it can be shown that the more rapid dilution is the result of an increased rate of synthesis of creatine and an increase in the total body creatine. This study affords direct evidence that methyltestosterone accelerates creatine synthesis.

When it is very difficult to synthesize an isotopic compound whose biologic turnover is to be studied, it may be possible to prepare the compound by biosynthesis and to study its turnover in the same or in another organism. An isotopic compound which is known to be a biologic precursor of the substance is administered to the subject and subsequently the substance is isolated serially and its isotope concentration is determined. In the case of substances in the dynamic state, the isotope concentration rises to a maximum shortly after the end of the administration of the isotopic precursor and then declines exponentially. The upward slope of the curve reflects the appearance of newly synthesized labeled molecules and their replacement of unlabeled molecules formed prior to the administration of the isotopic precursor. The declining portion of the curve results from the disappearance of labeled molecules and their replacement by newly synthesized molecules containing little or no isotope. Inasmuch as the degradation of the labeled molecules may in some cases result in isotopic fragments which can be reutilized for the synthesis of new molecules of the same substance, the rate of decline in isotope concentration may be slower than the actual rate of degradation. This discrepancy is probably not marked, however, for after the degradation of the labeled molecule, the isotopic fragment commonly enters a metabolic pool of similar fragments of lower isotope concentration. A representative sample of this pool is utilized for the synthesis of new molecules. These conditions approximate roughly the replacement of isotopic molecules by non-isotopic molecules and provide a good measure of the turnover rate of a substance in the dynamic state. This technique has been used to study the rates of turnover of serum and antibody proteins in the rabbit in whom it was found that the half lifetime of serum and antibody protein molecules is about two weeks (48). Shemin and Rittenberg have utilized this technique in an extensive study of the interrelationships involved in nitrogen transfer and of the rates of turnover of nitrogen in various tissues of the rat (49). 


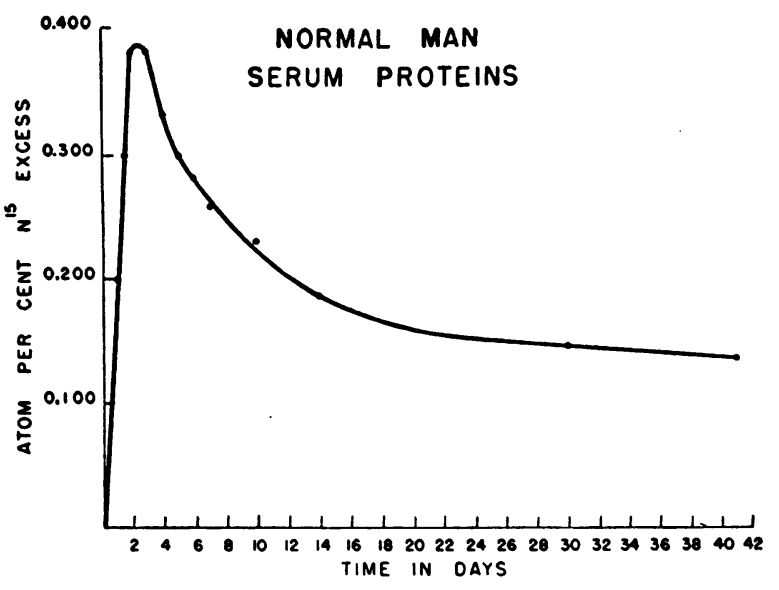

Fig. 1. N ${ }^{15}$ Concentration in Serum Proteins after Feeding N ${ }^{15}$ Labeled Glycine for Two Days

The kinetics of serum protein formation in the human have been investigated by this method (50). Glycine labeled with $\mathrm{N}^{15}$ was fed to a human subject for two days and the $\mathrm{N}^{15}$ concentrations in the total protein were determined. Figure 1 shows a curve representative of the data obtained in these studies. The maximal $\mathrm{N}^{15}$ concentration is reached shortly after the cessation of feeding labeled glycine and thereafter the isotope concentration declines in a roughly exponential manner. The $t_{1 / 2}$ value for total serum protein is about ten days.

With a $t_{1 / 2}$ of about ten days, the turnover time, $\bar{T}$, for a serum protein molecule is $t_{1 / 2} \times \ln 2$ or about 15 days. The normal human adult male with a total plasma volume of $45 \mathrm{ml}$. per kilogram of body weight has a serum protein content of about $3 \mathrm{Gms}$. per kilogram of body weight. Substitution in the equation $M=m \times \bar{T}$ yields a value for $m$ of $0.2 \mathrm{Gm}$. of serum protein per kilogram of body weight per day. In a normal man of 70 kilograms, approximately $14 \mathrm{Gms}$. of serum protein are synthesized and degraded daily. It should be remembered that $t_{1 / 2}$ values are approximations and are somewhat greater than the actual half lifetimes. Accordingly, the shorter actual half lifetimes will be reflected in proportionately greater rates of synthesis and degradation.

A theory and a practical procedure for evaluation of the rate of protein synthesis have recently been described by Sprinson and Rittenberg (51). A small amount of $\mathrm{N}^{15}$ labeled amino acid is administered to the subject and the $\mathrm{N}^{15}$ content in the urine excreted after the administration of the amino acid is determined. The greater the utilization of the amino acid for protein synthesis, the smaller the total excretion of $\mathrm{N}^{15}$ during the period of study. Mathematical treatment of the data on the cumulative excretion of $\mathrm{N}^{15}$ during a period of 48 to 72 hours after ingestion of the isotopic amino acid affords an estimation of the rate of protein synthesis and the size of the metabolic pool of nitrogen. The rate of protein synthesis per kilogram of body weight per day is approximately $0.2 \mathrm{Gm}$. of nitrogen in adult man. The nitrogen pool, i.e., the nitrogenous compounds which are derived from the diet or from the degradation of body constituents and which are utilized for the synthesis of tissue constituents, was found to be approximately $0.4-0.6 \mathrm{Gm}$. per kilogram in normal man. This procedure should prove to be valuable in the study of a variety of metabolic disorders and in the investigation of endocrine effects on nitrogen metabolism.

The rate of synthesis and degradation of a biologic substance can be determined by still another technique which measures the rate of incorporation of deuterium into the compound from the body fluids. This technique is applicable only to metabolic reactions in which hydrogen, or deuterium, is stably bound to carbon. By maintaining a steady deuterium concentration in the body fluids

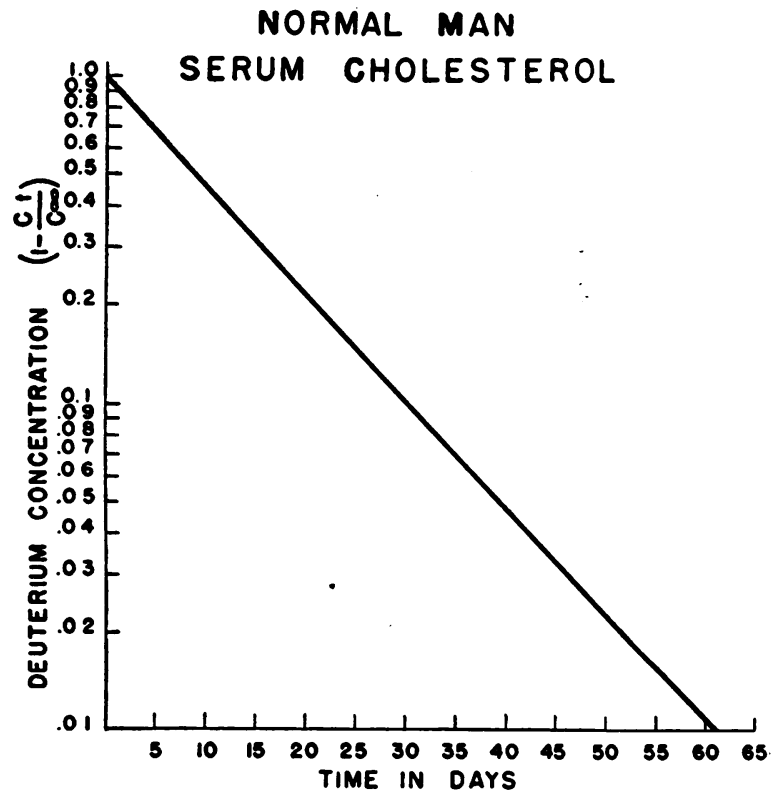

Fig. 2. Deuterium Concentration in Serum Cholesterol Ploteded Semilogarithmically

$C_{t}=$ deuterium concentration at any time $t$. $C_{\infty}=$ maximum deuterium concentration which is attained at infinite time. 
and by serial determinations of the deuterium concentration in the substance under study one can measure the rate at which the substance is synthesized. The maximum deuterium concentration which is achieved in this substance is usually lower than that of the body water since a fraction of the hydrogen atoms of most compounds is not derived from body water. The half lifetime of a substance is the time required to reach 50 per cent of this maximum concentration. Studies on the rate of formation of body cholesterol in the mouse have shown a half lifetime for the cholesterol molecule of 15 to 25 days (52). The rate of synthesis of serum cholesterol in a normal man has recently been investigated by this technique. Figure 2 describes a curve obtained by plotting the isotope concentration against time $\left[\log \left(1-\frac{\text { deuterium concentration at time } t}{\text { maximum deuterium concentration }}\right)\right.$ vs. time $]$.

The linear curve indicates that the change in isotope concentration is exponential in character and from the slope of the curve the half lifetime of serum cholesterol can be determined. In this normal male subject, the half lifetime is about nine days, the turnover time about 13 days (53).

\section{The Biosynthesis and Metabolism of Porphyrins}

The preceding discussion has been concerned with presenting examples of the main types of biological problems which are readily investigated with the aid of isotopes. These studies are for the most part unrelated and are diffusely spread over a number of fields of research. It may be well, therefore, to present an account of studies which illustrate the application of a variety of isotope methods to the investigation of a group of related problems in one field. The studies to be described are concerned with the biosynthesis and metabolism of porphyrins.

These studies were initiated with the finding by Shemin and Rittenberg that glycine is specifically utilized for the biologic formation of the protoporphyrin of hemoglobin (54). When glycine labeled with $\mathrm{N}^{15}$ is fed to a human, the hemin isolated from the red blood cells of the subject is found to contain $\mathrm{N}^{15}$ (55). Serial determinations of the $\mathrm{N}^{15}$ concentration in the hemin describe a curve (Figure 3$)(55,56)$ which is strikingly different from the type of curve of isotope con-

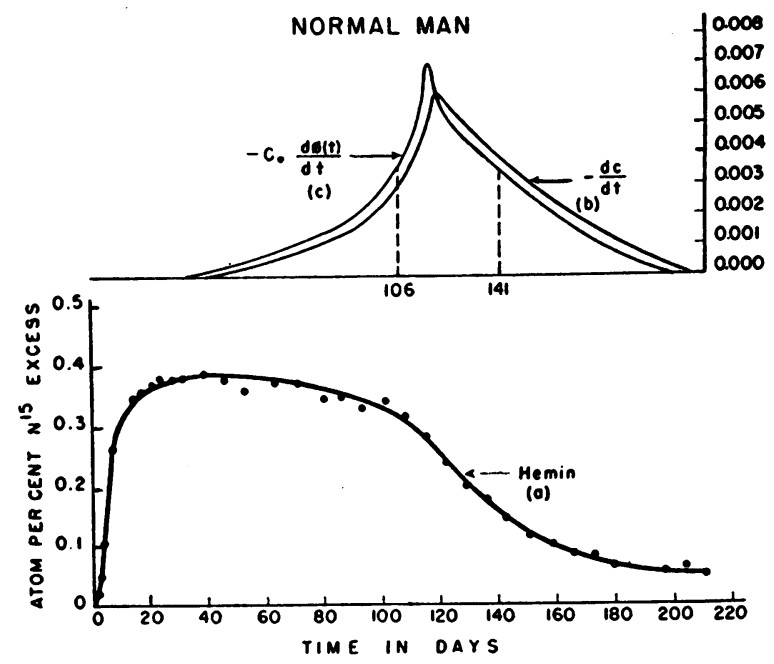

Fig. 3. N15 Concentration in Hemin After Feeding N15 Labeled Glycine for Two Days

centration observed in serum proteins after the administration of labeled glycine (Figure 1). The phenomenon observed in the case of serum proteins represents a dynamic process in which protein molecules are continuously being degraded and resynthesized. If hemin were in the dynamic state, even though the red cell itself were morphologically intact, the $\mathrm{N}^{15}$ concentration in the hemin would describe a similar curve. The curve of $\mathrm{N}^{15}$ concentration in hemin is fundamentally different, however, and cannot be the result of a random synthesis and degradation of hemoglobin in the peripheral blood. The presence of hemoglobin labeled with $\mathrm{N}^{15}$ in erythrocytes many weeks after the end of glycine administration must be due to synthesis and incorporation of hemoglobin labeled with $\mathrm{N}^{15}$ in the erythrocytes during formation of the cells in the bone marrow. The persistence of labeled hemoglobin in the red blood cells for months after its synthesis in the bone marrow indicates that once incorporated into the red cell the hemoglobin remains with the cell until the cell disintegrates. In brief, the persistence of the labeled hemoglobin reflects the survival or life span of the cells containing the hemoglobin.

The upward slope of the curve of isotope concentration in hemin represents the release into the circulation of erythrocytes containing isotopic hemoglobin and their replacement of cells formed prior to the administration of glycine which contain no isotopic hemoglobin. The maximum iso- 
tope concentration is reached and maintained when cells with heme of insignificant $\mathrm{N}^{15}$ concentration replace cells formed before the glycine administration. The declining portion of the curve reflects the destruction of cells containing labeled hemoglobin and the point of inflection represents the period of most marked destruction of these cells. The moderately abrupt decline indicates that the heme is not significantly, if at all, reutilized for new hemoglobin formation when the cell disintegrates. If the heme were reutilized significantly, a slower decline in isotope concentration would occur.

It is clear that normal human red blood cells are destroyed as a function of their age and not in indiscriminate fashion. The average life span of the human erythrocyte can be calculated from the curve of isotope concentration in hemin. These calculations are presented in detail elsewhere (55, 56). Values of 127,120 and 109 days have been obtained in two normal men and one normal woman. Since these subjects were in a steady state, these values correspond to the production
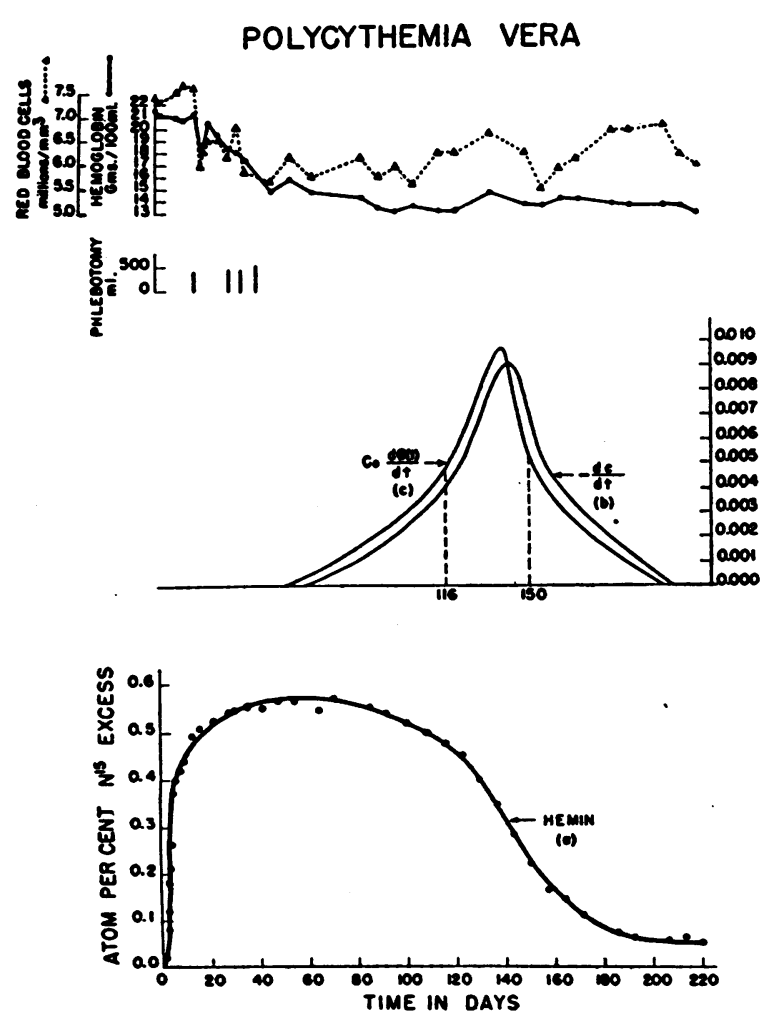

Fig. 4. N1s Concentration in Hemin Aftre Ferding N1s Labeled Glycine por Two Days and destruction of approximately 0.8 per cent of the red cells per day. Despite a rather wide range in the ages at death of the red cell population, the time span which encompasses the ages at death of half (the second and third quarters) of the cell population is relatively short. In the two normal male subjects this time span was found to be 28 and 35 days, in the normal female subject 32 days.

This isotope method possesses the unique advantage of making possible the study of the life span and pattern of destruction of the red cells in the same individual in whom the cells are made and destroyed without altering the usual state of the individual. Accordingly, it is well suited to the study of hemoglobin synthesis and red blood cell dynamics in normal and pathologic states.

Polycythemia vera is one of the clinical disorders which has been investigated with the aid of this method (56). The study was performed in an attempt to determine the factors which might be operative in the production of the characteristic abnormality of the disease, namely, the marked increase in the total number of circulating erythrocytes. Theoretically this increase might result from one or both of these factors: (1) an increased rate of hemoglobin and erythrocyte synthesis and (2) prolonged life of the erythrocytes. A patient in a fully developed stage of the disease was given glycine labeled with $\mathrm{N}^{15}$ in a dosage equivalent to that used in the normal subjects. Figure 4 describes the curve of the isotope concentration in hemin. The pattern of red cell destruction is normal and the average life span of the red cells is 131 days, a value close to the values obtained in the normal subjects. Inasmuch as the erythrocyte life span is essentially normal, the marked increase in the number of circulating red cells must be associated with an elevated rate of red cell and hemoglobin synthesis. In this patient the rate of synthesis was 2.5 times the normal value.

These findings demonstrate a functional hyperactivity of the blood-forming apparatus in polycythemia vera in a fully developed stage of the disease. It is likely that the development of the polycythemia earlier in the disease is also characterized by increased hematopoietic activity with the maintenance of a normal erythrocyte life span. Various theories have been proposed for the etiology of polycythemia vera but conclusive 
proof in support of any of them is lacking. The fundamental cause of the functional hyperactivity of hematopoietic tissue remains unknown. The theory, first proposed by Minot and Buckman (57), that polycythemia vera is a neoplasm, appears to be most compatible with available evidence. The persistent bone marrow hyperplasia involving all marrow elements, the development of leukemia in some cases of polycythemia vera and the development of polycythemia in some cases of leukemia suggest that this is a neoplastic process. It appears to be a benign neoplasm which can develop malignant characteristics. If it is a neoplasm, it is an instance of neoplastic growth which is associated with an increase in synthetic activity with no apparent diminution in degradative activity. The fundamental cause, however, remains no less obscure than that of other forms of neoplasia.

Sickle cell anemia is another clinical disorder which has been investigated with the aid of the isotope method (56). A patient with the characteristic findings of this disease received labeled glycine in a dosage similar to that used in the normal subjects. The curve of isotope concentration in the hemin is shown in Figure 5. It is markedly different from the normal. Following a rapid rise to a maximum on the seventh day, the curve declines exponentially. The exponential decline results from a random disappearance of labeled heme from the circulating blood, i.e., the heme is removed from the circulation at a rate which is independent of the age of the heme at the time of its degradation. A curve of this shape could result from (1) a random destruction of the

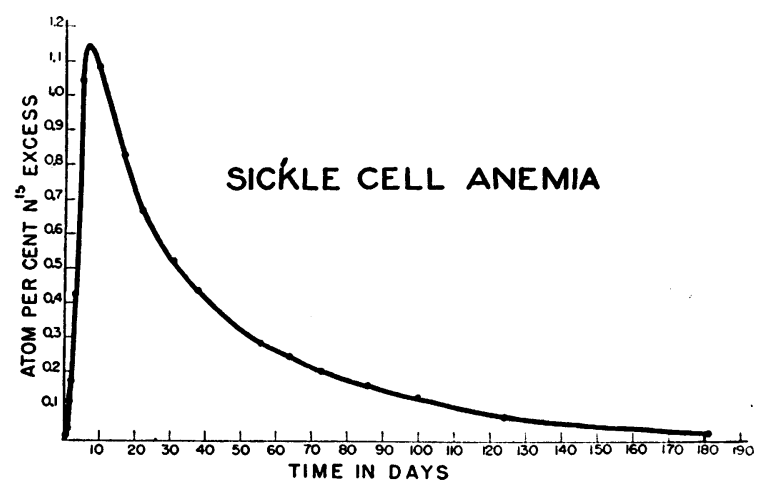

Fig. 5. N15 Concentration in Hemin After Feeding N15 Labeled Glycine for Two Days red blood cells and a consequent loss of labeled heme from the circulating blood; or (2) a random degradation and synthesis of heme in circulating red blood cells which are morphologically intact; or (3) random synthesis and degradation of heme in red blood cells which are themselves undergoing random destruction. The latter two possibilities seemed unlikely in the light of earlier studies which demonstrated that the hemoglobin of circulating erythrocytes is not in the dynamic state. However, when the whole blood of patients with sickle cell anemia is incubated with $\mathrm{N}^{15}$ labeled glycine heme labeled with $\mathrm{N}^{15}$ is formed (58). This in vitro synthesis of heme indicates that the peripheral blood in patients with sickle cell anemia may synthesize heme. The synthesis in the in vitro experiments occurs at a rate of only 0.1 -0.2 per cent of the red cell heme in 24 hours. If all the hemoglobin in the circulating erythrocytes of sickle cell anemia were synthesized in the peripheral blood at a rate of the same order of magnitude as in the in vitro experiments, the hemoglobin turnover would be ten to 25 times slower than that which is actually observed in this case. It would seem that the random disappearance of heme must be due for the most part to an indiscriminate destruction of erythrocytes. If some random synthesis of heme in the peripheral blood of patients with sickle cell anemia does occur, it probably plays a very minor role in the hemoglobin turnover in this disease.

Inasmuch as the erythrocytes of sickle cell anemia are destroyed indiscriminately, they do not have a true life span and their survival is more appropriately measured in terms of their half lifetime. The half lifetime $\left(t_{1 / 2}\right)$, i.e., the time required for the isotope concentration to decline from any given value on the declining portion of the curve to one-half that value, is 29 days in this case. The mean survival time, or turnover time $\left(t_{1 / 2} \times \ln 2\right)$, is 42 days. With these data and determinations of total circulating hemoglobin and red blood cell volumes, the rates of hemoglobin and red cell production may be calculated. The rates in this patient were found to be nearly three times the normal.

The increased hematopoietic activity is most likely a compensatory response to the markedly reduced number of erythrocytes. The diminished survival time reflects a defect which is in all prob- 
ability intrinsic to the red cell. This defect, which probably involves the structure of the red cell membrane, may be associated with the sickling process but cannot be ascribed to this phenomenon alone, since the red cells of individuals with sickle cell trait but without anemia are not abnormally susceptible to destruction $(59,60)$.

Pernicious anemia is another disease process which has been investigated (56). A patient with the typical physical and laboratory findings of the disease received $\mathrm{N}^{15}$ labeled glycine. No antianemia therapy had been given when the experiment was begun. The curve of isotope concentration in hemin, the red blood cell counts, hemoglobin values, and reticulocyte counts during the course of the study are shown in Figure 6. The isotope concentration in hemin rose rapidly and was approaching its maximum on the 16th day. It was considered inadvisable to withhold treatment longer and liver extract therapy in large dosage was begun. A satisfactory reticulocyte response and rise in hemoglobin and red blood cell counts occurred.

The rise in hemoglobin and erythrocyte levels was accompanied by a decline in the isotope concentration in the hemin. This decline was anticipated for a dilution of the isotope concentration in the hemin of circulating erythrocytes should result from the release into the circulation of large numbers of new cells formed when the isotope concentration in the body glycine had fallen to a low value. The decline in isotope concentration persisted, however, after the erythrocyte and hemoglobin values approached normal levels and a mere dilution effect should have been minimal. To differentiate a dilution effect from actual destruction of the cells containing labeled hemoglobin, the changes in the total amount of $\mathrm{N}^{15}$ in the heme of circulating erythrocytes were determined. It has been shown (61) that the hemo-

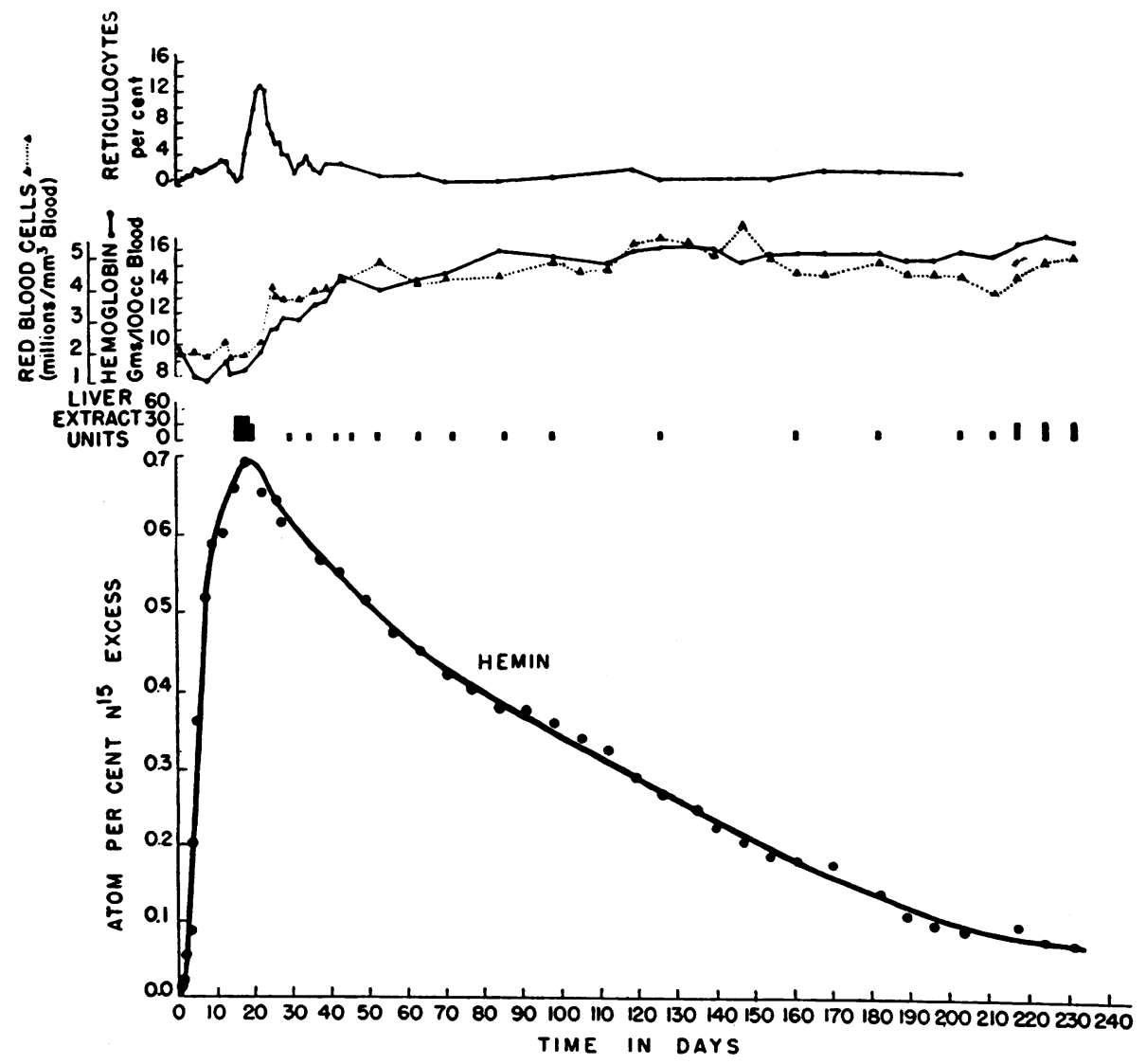

Fig. 6. N15 Concentration in Hemin after Feeding N15 Labeled Glycine for two Days to a Subject with Pernicious Anemia 


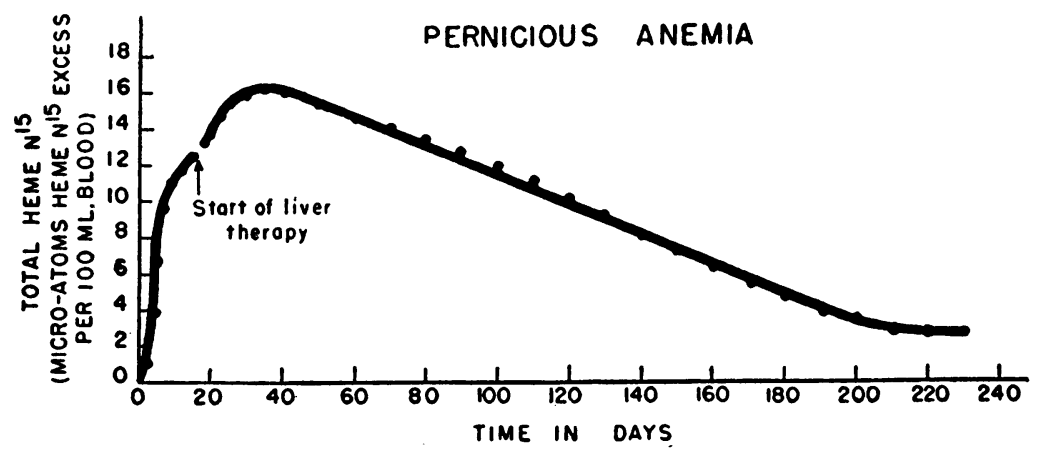

Fig. 7. Total Heme ${ }^{15}$ after Feeding N15 Labeled Glycine for Two Days

globin concentration in the peripheral blood serves as a reliable index of the total hemoglobin in circulation before and after the start of liver treatment in pernicious anemia. Accordingly, a curve representing the changes in the total amount of heme $\mathrm{N}^{15}$ in circulation could be obtained by multiplying the hemoglobin concentration in the peripheral blood by the isotope concentration in the hemin (Figure 7).

The initial rise in total heme $\mathrm{N}^{15}$ is similar to the rise in isotope concentration noted in Figure 6. After the start of liver therapy, an additional rise occurs due to the influx of many new cells. Although these newly formed cells contain heme of relatively low isotope concentration, their total number represents a considerable increment in the quantity of heme $\mathrm{N}^{15}$ in the circulating blood. The maximum value for total heme $\mathrm{N}^{15}$ is attained about two weeks after the start of liver therapy. If the cells of untreated pernicious anemia enjoyed a normal life span, the curve would have maintained a plateau until the 40th to 60th day and then would have begun to decline. The curve declines, however, in linear fashion almost immediately after reaching its peak. If all the cells were destroyed indiscriminately, the decline would have been exponential. The linear decline suggests that the cell population is mixed, many of the cells being destroyed indiscriminately and others as a function of their age. From the declining portion of the curve it is possible to calculate that the mean survival time of the mixed cell population is 90 days and that the mean survival time of the cells formed prior to liver therapy is approximately 85 days. These calculations are presented in detail elsewhere (56).
With these data and determinations of total hemoglobin and red blood cell values, it can be shown that the rate of production of erythrocytes capable of reaching the peripheral blood is only about 50 per cent of normal. This diminished rate of production and the diminished survival time of the cells in circulation are consistent with the view that in untreated pernicious anemia the erythrocytes are intrinsically defective. The absence of an abnormal hemolytic factor in the plasma of pernicious anemia patients is supported by studies with the Ashby technique $(62,63)$ which have shown an essentially normal survival of normal cells transfused to recipients with pernicious anemia. There is no marked deficiency in the rate of production of circulating hemoglobin which in this case was 80 per cent of normal.

After treatment with liver extract for one year, the patient was again studied. The findings reveal that the red blood cells are destroyed as a function of age, not indiscriminately, and that their average life span'is 129 days, a normal value. Complete restoration to normal red cell dynamics has occurred (Figure 8).

In the untreated state, this patient showed only mild diminution in the rate of production of circulating hemoglobin. But even a normal rate of production and destruction of circulating red cell hemoglobin fails to provide an adequate explanation for the very large amounts of bile pigment which are produced in pernicious anemia. To investigate this and other problems of bile pigment metabolism, the biologic origin of bile pigment has been studied with the isotope technique.

It has commonly been assumed that bile pigment normally is derived almost exclusively, if not com- 
pletely, from the degradation of hemoglobin of mature circulating red blood cells. In accordance with this assumption, the appearance of $\mathrm{N}^{15}$ in the bile pigment following the administration of $\mathrm{N}^{15}$ labeled glycine should reflect the destruction of red blood cells containing labeled hemoglobin. Since in the normal human no significant number of red cells is destroyed for several weeks after their release into the circulation, there should be no significant concentration of $\mathrm{N}^{15}$ in bile pigment during the early part of the experiments in the normal subjects. Stercobilin isolated from the stools collected during the first eight days of the experiment in one of the normal subjects was found, however, to have a high concentration of $\mathrm{N}^{15}$ (64). This finding suggests that a portion of bile pigment is derived from one or more of the following sources: (1) hemoglobin of red blood cells which are destroyed shortly after reaching the peripheral blood or never reach it and are destroyed in the bone marrow; (2) porphyrins which are not utilized for hemoglobin production; or (3) direct synthesis of bile pigment via a pathway which does not involve degradation of a porphyrin ring. From quantitative considerations it appears unlikely that myoglobin or the respiratory heme pigments serve as a significant source of this portion of bile pigment.

The finding of an additional source of bile pigment formation appeared to offer a reasonable explanation for the discrepancy in untreated pernicious anemia between the very high levels of bile

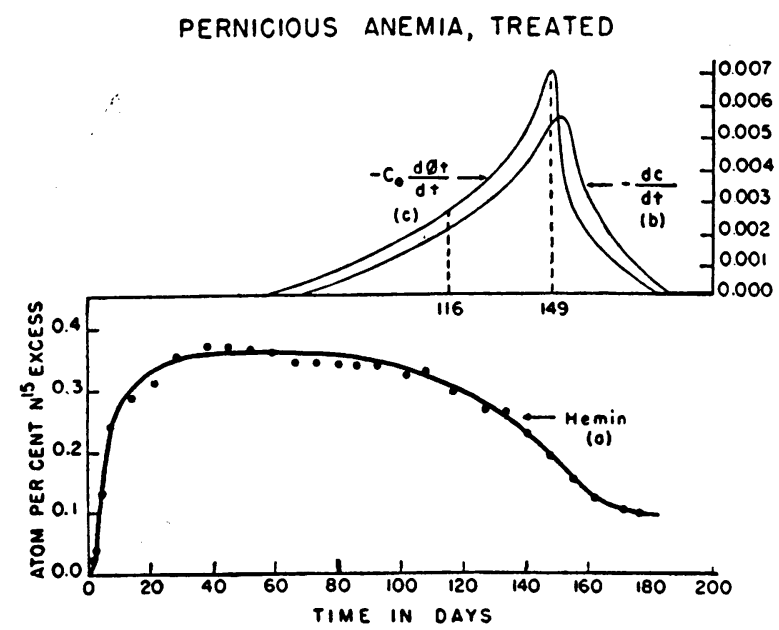

Fig. 8. N15 Concentration in Hemin after Feeding N15 Labeled Glycine for Two Days pigment production and the relatively moderate destruction of circulating red cell hemoglobin. When a similar study was performed in the patient with pernicious anemia, an extraordinarily high concentration of $\mathrm{N}^{15}$ was found in the stercobilin isolated during the first several days of the experiment (65). These findings indicate that a very large portion of the bile pigment produced in this disease is derived from one or more of the additional sources which have been suggested.

The investigation of porphyrin metabolism was facilitated by the finding that heme synthesis occurs in vitro in the blood of patients with sickle cell anemia (58) and in avian nucleated erythrocytes (66). Since significant heme synthesis is not observed in normal human blood under similar experimental conditions, the in vitro synthesis observed in the blood of sickle cell anemia was believed to be due to the presence of numerous immature reticulated cells. On incubation of blood samples from other subjects with hematologic disorders characterized by elevated reticulocyte counts, however, significant heme synthesis was not observed (58). These findings indicated that although the reticulocytes in the blood of sickle cell anemia might be responsible for the heme synthesis, the mere presence of numerous reticulocytes in the blood of subjects with other hematologic disorders does not insure the ability of such blood to synthesize heme in vitro. Further studies (67) have confirmed the finding that some blood samples with elevated reticulocyte counts from patients with other blood dyscrasias do not perform significant in vitro heme synthesis. These studies have shown, however, that other blood samples with elevated reticulocyte counts from subjects with disorders other than sickle cell anemia may synthesize heme in vitro. To investigate some of the problems posed by these observations, the capacity for heme synthesis of reticulocytes in the blood of experimental animals which had no disorder of erythrocyte or hemoglobin formation was studied. The blood of previously normal rabbits in whom reticulocytosis was induced by bleeding or phenylhydrazine hemolysis was incubated aerobically with $\mathrm{N}^{15}$ labeled glycine for 24 hours. The $\mathrm{N}^{15}$ concentrations in the hemin of these blood samples revealed that approximately $0.3-0.9$ per cent of the total heme in the in vitro system was 
newly synthesized during the 24 hour incubation period. These findings demonstrate that mammalian reticulocytes possess a significant capacity for heme synthesis and suggest that some heme synthesis may occur in vivo in the reticulocyte after its release into the peripheral blood.

The in vitro synthesis of heme by avian erythrocytes has been utilized to determine the precursors which participate in the biosynthesis of protoporphyrin. It has been found, using $\mathrm{C}^{14}$ labeled compounds, that both carbon atoms of acetic acid, the carbonyl carbon of pyruvic acid, and the methylene carbon of glycine are utilized in the synthesis of the carbon skeleton of protoporphyrin (68). The utilization of the amino group of glycine for the synthesis of both types of pyrrole rings of protoporphyrin has been demonstrated by finding equal $\mathrm{N}^{15}$ concentrations in the two pyrrole types which were obtained by appropriate degradation of hemin formed following $\mathrm{N}^{15}$ glycine feeding (69).

The biosynthesis of porphyrins and bile pigment has been investigated in a patient with congenital porphyria who excretes large amounts of uroporphyrin I and coproporphyrin I. Shortly after the feeding of $\mathrm{N}^{15}$ labeled glycine, very high concentrations of $\mathrm{N}^{15}$ were found in the coproporphyrin I, uroporphyrin I and stercobilin (65). These findings indicate that glycine is specifically utilized in the biosynthesis of porphyrins of the I isomer configuration as well as of protoporphyrin IX which is of the etioporphyrin III isomer configuration. They furnish additional evidence in support of earlier observations (64) that bile pigment is derived in part from one or more sources other than the hemoglobin of mature circulating erythrocytes.

\section{CONCLUSION}

Despite the relatively short period of time during which isotopes have been available, tracer techniques have exerted a pervasive influence on biochemical thought and practice. A definitive evaluation of this influence may have to await more extensive development of isotope investigation. It is possible, however, to indicate the two principal developments in biochemical thought which have already occurred and are due in part to the impact of tracer techniques.
Tracer studies have helped to develop a concept which provides insight into the mechanisms of biological synthesis. It has become evident that complex organic substances to a large extent are synthesized not from compounds of similar complex configuration but rather from small compounds of simple structure. The extensive participation of glycine and acetic acid in biosynthetic processes has focussed attention on the biochemical versatility and significance of small simple compounds.

The outstanding contribution of isotope methodology to biochemical thinking has been the development of the concept of the dynamic state of the body constituents. The experimental basis and theoretical implications of this concept, which includes an appreciation of the kinetics of biochemical reactions, represent a major advance toward a thorough understanding of the processes which occur in the living cell.

\section{BIBLIOGRAPHY}

1. Buchanan, J. M., and Hastings, A. B., The use of isotopically marked carbon in the study of intermediary metabolism. Physiol. Rev., 1946, 26, 120.

2. Kamen, M. D., Radioactive Tracers in Biology. Academic Press, New York, 1947.

3. Hevesy, G., Radioactive Indicators; Their Application in Biochemistry, Animal Physiology, and Pathology. Interscience Publishers, New York, 1948.

4. A Symposium on the Use of Isotopes in Biology and Medicine. University of Wisconsin Press, Madison, 1948.

5. Preparation and measurement of isotopes and some of their medical aspects. Supplement to the U. S. Naval Medical Bulletin, March-April, 1948.

6. Lawrence, J. H., and Hamilton, J. G., Advances in Biological and Medical Physics. Academic Press, New York, 1948, Vol. 1.

7. Biological Applications of Tracer Elements. (Cold Spring Harbor Symposium on Quantitative Biology.) Long Island Biological Laboratory, Cold Spring Harbor, Long Island, N. Y., 1948, 13.

8. Hevesy, G., and Hofer, E., Elimination of water from the human body. Nature, 1934, 134, 879.

9. Moore, F. D., Determination of total body water and solids with isotopes. Science, 1946, 104, 157.

10. Gellhorn, A., and Flexner, L. B., Transfer of water aross the placenta of the guinea pig. Am. J. Physiol., 1942, 136, 750.

11. Ussing, H. H., Analysis of protein by means of deuterium-containing amino-acids. Nature, 1939, 144, 977. 
12. Rittenberg, D., and Foster, G. L.; A new procedure for quantitative analysis by isotope dilution, with application to determination of amino acids and fatty acids. J. Biol. Chem., 1940, 133, 737.

13. Gest, H., Kamen, M. D., and Reiner, J. M., The theory of isotope dilution. Arch. Biochem., 1947, $12,273$.

14. Shemin, D., and Foster, G. L., The isotope dilution method of amino acid analysis. Ann. New York Acad. Sc., 1946, 47, 119.

15. Foster, G. L., Some amino acid analyses of hemoglobin and $\beta$-lactoglobulin. J. Biol. Chem., 1945, $159,431$.

16. Shemin, D., Amino acid determinations on crystalline bovine and human serum albumin by isotope dilution method. J. Biol. Chem., 1945, 159, 439.

17. Graff, S., Rittenberg, D., and Foster, G. L., The glutamic acid of malignant tumors. J. Biol. Chem., 1940, 133, 745.

18. Keston, A. S., Udenfriend, S., and Cannan, R. K., Micro-analysis of mixtures (amino acids) in the form of isotopic derivatives. J. Am. Chem. Soc., 1946, 68, 1390.

19. Bloch, K., and Anker, H., An extension of the isotope dilution method. Science, 1948, 107, 228.

20. Bloch, K., and Schoenheimer, R., Studies in protein metabolism; the metabolic relation of creatine and creatinine studied with isotopic nitrogen. J. Biol. Chem., 1939, 131, 111.

21. Brand, E., Harris, M. M., Sandberg, M., and Ringer, A. I., Studies on the origin of creatine. Am. J. Physiol., 1929, 90, 296.

22. Brand, E., and Harris, M. M., Some aspects of intermediary protein metabolism. Science, 1933, 77, 589.

23. Fisher, R. B., and Wilhelmi, A. E., The Metabolism of Creatine. II. The conversion of arginine into creatine in the isolated rabbit heart. Biochem. J., 1937, 31, 1136.

24. Borsook, H., and Dubnoff, J.' W., The formation of creatine from glycocyamine in the liver. J. Biol. Chem., 1940, 132, 559.

25. Bloch, K., and Schoenheimer, R., The biological formation of creatine. J. Biol. Chem., 1940, 133, 633.

26. Bloch, K., and Schoenheimer, R., The biological demethylation of sarcosine to glycine. J. Biol. Chem, 1940, 135, 99.

27. Borsook, H., and Dubnoff, J. W., The synthesis of glycocyamine in rat kidney and a mechanism of creatine synthesis in vivo. Science, 1940, 91, 551.

28. Bloch, K., and Schoenheimer, R., The biological origin of the amidine group in creatine. J. Biol. Chem., 1940, 134, 785.

29. Bloch, K., and Schoenheimer, R., The biological precursors of creatine. J. Biol. Chem., 1941, 138, 167.

30. du Vigneaud, V., Chandler, J. P., Cohn, M., and Brown, G. B., The transfer of the methyl group from methionine to choline and creatine. J. Biol. Chem., 1940, 134, 787.

31. du Vigneaud, V., Cohn, M., Chandler, J. P., Schenk, J. R., and Simmonds, S., The utilization of the methyl group of methionine in the biological synthesis of choline and creatine. J. Biol. Chem., 1941, 140, 625.

32. Sonne, J. C., Buchanan, J. M., and Delluva, A. M., Biological precursors of uric acid carbon. J. Biol. Chem., 1946, 66, 395.

33. Sonne, J. C., Buchanan, J. M., and Delluva, A. M., Biological precursors of uric acid. I. The role of lactate, acetate, and formate in the synthesis of the ureide groups of uric acid. J. Biol. Chem., 1948, 173, 69.

34. Buchanan, J. M., Sonne, J. C., and Delluva, A. M., Biological precursors of uric acid. II. The role of lactate, glycine, and carbon dioxide as precursors of the carbon chain and nitrogen atom 7 of uric acid. J. Biol. Chem., 1948, 173, 81.

35. Shemin, D., and Rittenberg, D., On the utilization of glycine for uric acid synthesis in man. J. Biol. Chem., 1947, 167, 875.

36. Karlsson, J. L., and Barker, H. A., Biosynthesis of uric acid labeled with radioactive carbon. J. Biol. Chem., 1949, 177, 597.

37. Wood, H. G., Tracer studies on the intermediary metabolism of carbohydrates, in: A Symposium on the Use of Isotopes in Biology and Medicine. University of Wisconsin Press, Madison, 1948, p. 209.

38. Evans, E. A., Jr., The fixation of $\mathrm{CO}$, by animal tissues. The Harvey Lect., 1943-44, 39, 273.

39. Gurin, S., and Crandall, D. I., The biological oxidation of fatty acids, in: Cold Spring Harbor Symposium on Quantitative Biology. Long Island Biological Laboratory, Cold Spring Harbor, Long Island, N. Y., 1948, 13, 118.

40. Stetten, D., Jr., Biological relationships of choline, ethanolamine, and related compounds. J. Biol. Chem., 1941, 140, 143.

41. Weinhouse, S., Medes, G., and Floyd, N. F., Fatty acid metabolism. The mechanism of ketone body synthesis from fatty acids, with isotopic carbon as tracer. J. Biol. Chem., 1944, 155, 143.

42. Zilversmit, D. B., Entenman, C., and Fishler, M. C., On the calculation of "turnover time" and "turnover rate" from experiments involving the use of labelling agents. J. Gen. Physiol., 1943, 26, 325.

43. Branson, $H$., The use of isotopes in an integral equation description of metabolizing systems, in: Cold Spring Harbor Symposium on Quantitative Biology. Long Island Biological Laboratory, Cold Spring Harbor, Long Island, N. Y., 1948, 13, 35.

44. Radin, N., Isotope techniques in biochemistry. Nucleonics, 1948, 2, 50.

45. Bloch, K., Schoenheimer, R., and Rittenberg, D., Rate of formation and disappearance of body creatine in normal animals. J. Biol. Chem., 1941, 138, 155. 
46. Hoberman, H. D., Sims, E. A. H., and Peters, J. H., Creatine and creatinine metabolism in the normal male adult studied with the aid of isotopic nitrogen. J. Biol. Chem., 1948, 172, 45.

47. Hoberman, H. D., Sims, E. A. H., and Engstrom, W. W., The effect of methyltestosterone on the rate of synthesis of creatine. J. Biol. Chem., 1948, 173, 111.

48. Schoenheimer, R., Ratner, S., Rittenberg, D., and Heidelberger, M., The interaction of antibody protein with dietary nitrogen in actively immunized animals. J. Biol. Chem., 1942, 144, 545.

49. Shemin, D., and Rittenberg, D., Some interrelationships in general nitrogen metabolism. J. Biol. Chem., 1944, 153, 401.

50. Unpublished data from this laboratory.

51. Sprinson, D., and Rittenberg, D., The rate of interaction of the amino acids of the diet with the tissue proteins. J. Biol. Chem., in press.

52. Rittenberg, D., and Schoenheimer, R., Deuterium as an indicator in the study of intermediary metabolism. XI. Further studies on the biological uptake of deuterium into organic substances, with special reference to fat and cholesterol formation. J. Biol. Chem., 1937, 121, 235.

53. London, I. M., and Rittenberg, D., Unpublished data.

54. Shemin, D., and Rittenberg, D., The biological utilization of glycine for the synthesis of the protoporphyrin of hemoglobin. J. Biol. Chem., 1946, 166, 621.

55. Shemin, D., and Rittenberg, D., The life span of the human red blood cell. J. Biol. Chem., 1946, 166, 627.

56. London, I. M., Shemin, D., West, R., and Rittenberg, D., Heme synthesis and red blood cell dynamics in normal humans and in subjects with polycythemia vera, sickle-cell anemia, and pernicious anemia. J. Biol. Chem., 1949, 179, 463.

57. Minot, G. R., and Buckman, T. E., Erythremia (polycythemia rubra vera). The development of anemia; the relation to leukemia; consideration of the basal metabolism, blood formation and destruction and fragility of the red cells. Am. J. M. Sc., 1923, 166, 469.
58. London, I. M., Shemin, D., and Rittenberg, D., The in vitro synthesis of heme in the human red blood cell of sickle cell anemia. J. Biol. Chem., 1948, 173, 797.

59. Singer, K., Robin, S., King, J. C., and Jefferson, R. N., The life span of the sickle cell and the pathogenesis of sickle cell anemia. J. Lab. \& Clin. Med., 1948, 33, 975.

60. Callender, S. T. E., Nickel, J. F., and Moore, C. V., Sickle cell disease: studied by measuring the survival of transfused red blood cells. J. Lab. \& Clin. Med., 1949, 34, 90.

61. Gibson, J. G., 2nd, Clinical studies of the blood volume. VI. Changes in blood volume in pernicious anemia in relation to the hematopoietic response to intramuscular liver extract therapy. J. Clin. Invest., 1939, 18, 401.

62. Loutit, J. F., The life and death of the red corpuscle. Proc. Roy. Soc. Med., 1946, 39, 757.

63. Mollison, P. L., Survival of transfused erythrocytes, with special reference to cases of acquired hæmolytic anæmia. Clin Sc., 1947, 6, 137.

64. London, I. M., West, R., Shemin, D., and Rittenberg $\mathrm{D}$., On the origin of stercobilin in humans. Federation Proc., 1948, 7, 169.

65. London, I. M., Shemin, D., West, R., and Rittenberg, D., Unpublished data.

66. Shemin, D., London, I. M., and Rittenberg, D., The in vitro synthesis of heme from glycine by the nucleated red blood cell. J. Biol. Chem., 1948, 173, 799.

67. London, I. M., Shemin, D., and Rittenberg, D., Studies in hemoglobin formation with the aid of the isotope technique. J. Clin. Invest., 1949, 28, 796.

68. Radin, N. S., Rittenberg, D., and Shemin, D., Biosynthesis of heme. Federation Proc., 1949, 8, 240.

69. Wittenberg, .J., and Shemin, D., The utilization of glycine for the biosynthesis of both types of pyrroles in protoporphyrin. J. Biol. Chem., 1949, 178, 47. 